\title{
Repair of a traumatic subarachnoid-pleural fistula with the percutaneous injection of fibrin glue in a 2-year-old
}

\author{
Jason K. Chu, MD, MSc, ${ }^{1}$ Brandon A. Miller, MD, PhD, ${ }^{1}$ Michael P. Bazylewicz, MD, ${ }^{2}$ \\ John F. Holbrook, MD, ${ }^{2}$ and Joshua J. Chern, MD, PhD ${ }^{3}$ \\ Departments of ${ }^{1}$ Neurosurgery and ${ }^{2}$ Radiology, Emory University School of Medicine, Atlanta; and ${ }^{3}$ Pediatric Neurosurgery \\ Associates, Children's Healthcare of Atlanta, Georgia
}

\begin{abstract}
Subarachnoid-pleural fistulas (SPFs) are rare clinical entities that occur after severe thoracic trauma or iatrogenic injury during anterolateral approaches to the spine. Treatment of these fistulas often entails open repair of the dural defect. The authors present the case of an SPF in a 2-year-old female after a penetrating injury to the chest. The diagnosis of an SPF was suspected given the high chest tube output and was confirmed with a positive $\beta 2$-transferrin test of the chest tube fluid, as well as visualization of dural defects on MRI. The dural defects were successfully repaired with CT-guided percutaneous epidural injection of fibrin glue alone. This case represents the youngest pediatric patient with a traumatic SPF to be treated percutaneously. This technique can be safely used in pediatric patients, offers several advantages over open surgical repair, and could be considered as an alternative first-line therapy for the obliteration of SPFs.
\end{abstract} http://thejns.org/doi/abs/10.3171/2015.5.PEDS15194

KEY WORDS subarachnoid-pleural fistula; fibrin glue; gunshot wound; spinal penetrating injury; pediatrics; technique; trauma; spine

$\mathrm{T}$ HE first case of a subarachnoid-pleural fistula (SPF) after a gunshot wound to the chest was reported by Milloy et al. in $1959 .{ }^{15}$ Over the last 60 years, the literature on SPF has been sparse, although its 2 most commonly reported causes are thoracic trauma and iatrogenic injury during anterolateral approaches to the spine. Documented cases of pediatric SPF are even rarer. Nonetheless, there is consensus that an anomalous connection between the subarachnoid and pleural spaces is not likely to resolve spontaneously and requires surgical intervention to repair the dural defect. Here, we present the youngest pediatric case of SPF secondary to penetrating trauma, as well as the first report of successful fistula obliteration with CTguided percutaneous epidural injection of fibrin glue over the site of the CSF leak.

\section{Case Report}

History and Examination

A previously healthy 2-year-old female was an unfortunate bystander to a domestic dispute and suffered a through-and-through gunshot wound to her left upper chest. An entry wound was identified in her left midclavicular line approximately $3 \mathrm{~cm}$ medial and superior to the nipple, and an exit wound was seen in the midline of her upper thoracic region. She was intubated and a left-sided chest tube was placed for the treatment of a left hemopneumothorax and returned serosanguineous fluid. Neurological exam was significant for bilateral lower extremity plegia, a T-3 sensory level, and absent rectal tone, consistent with an American Spinal Injury Association (ASIA) Grade 
A spinal cord injury at T-3. Computed tomography scanning revealed extensive left-sided pulmonary contusions without mediastinal injury, as well as a left-sided comminuted pedicle and laminar fractures at T-3 with several osseous fragments within the lateral aspect of the spinal canal. There was no significant spinal canal stenosis (Fig. 1). Magnetic resonance imaging of the spine demonstrated intramedullary $\mathrm{T} 2$ hyperintensity at the level of T-2 and $\mathrm{T}-3$, indicative of spinal cord contusion. Additionally, a subdural hygroma was seen at the lower thoracic and upper lumbar levels with anterior displacement of her lower thoracic cord, conus medullaris, and cauda equina (Fig. 2).

\section{Treatment}

She received a blood transfusion for anemia, was started on norepinephrine to maintain perfusion pressure, and was transferred to the pediatric intensive care unit. Her chest tube was set to suction for 24 hours.

Spinal stabilization was not deemed immediately necessary. The patient was weaned from pressors, was successfully extubated, and had her chest tube set to water seal on hospital Day 2. On hospital Day 3, she was noted to have increased serous output from her chest tube $(274 \mathrm{ml}$ over 24 hours), and a sample tested positive for $\beta 2$-transferrin. She was transferred to the floor in stable condition on hospital Day 4. Between hospital Days 4 and 7, she was kept on bed rest but continued to have high serous output from her chest tube (range 50-230 ml over 24 hours). On hospital Day 8, her chest tube became dislodged and was removed. A subsequent thoracic ultrasound on Day 9 revealed a large pleural effusion. She remained asymptomatic but was noted to have enlargement of her pleural effusion on serial chest radiographs. On hospital Day 10, thoracic MRI with constructive interference in steady state (CISS) sequence was performed, demonstrating a right posterolateral dural defect at the level of T-2 and left lateral dural defect at the level of T-3 (Fig. 3). A 12-Fr chest tube was reinserted on the same day, with the return of a large amount of clear fluid. Over the next 24 hours, the chest tube put out $272 \mathrm{ml}$ of serous fluid. She underwent CT-guided percutaneous epidural injection of $2 \mathrm{ml}$

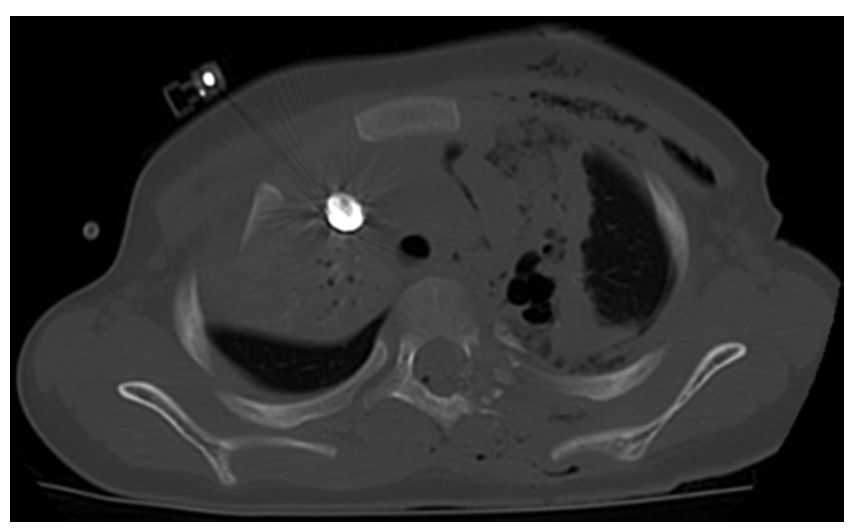

FIG. 1. Axial CT scan of the thorax demonstrating a penetrating ballistic injury to the left chest with associated extensive left upper lobe contusion and chest tube placement. There are comminuted ballistic fractures of the left pedicle and lamina of T-3 with several small osseous fragments protruding into the left aspect of the spinal canal.
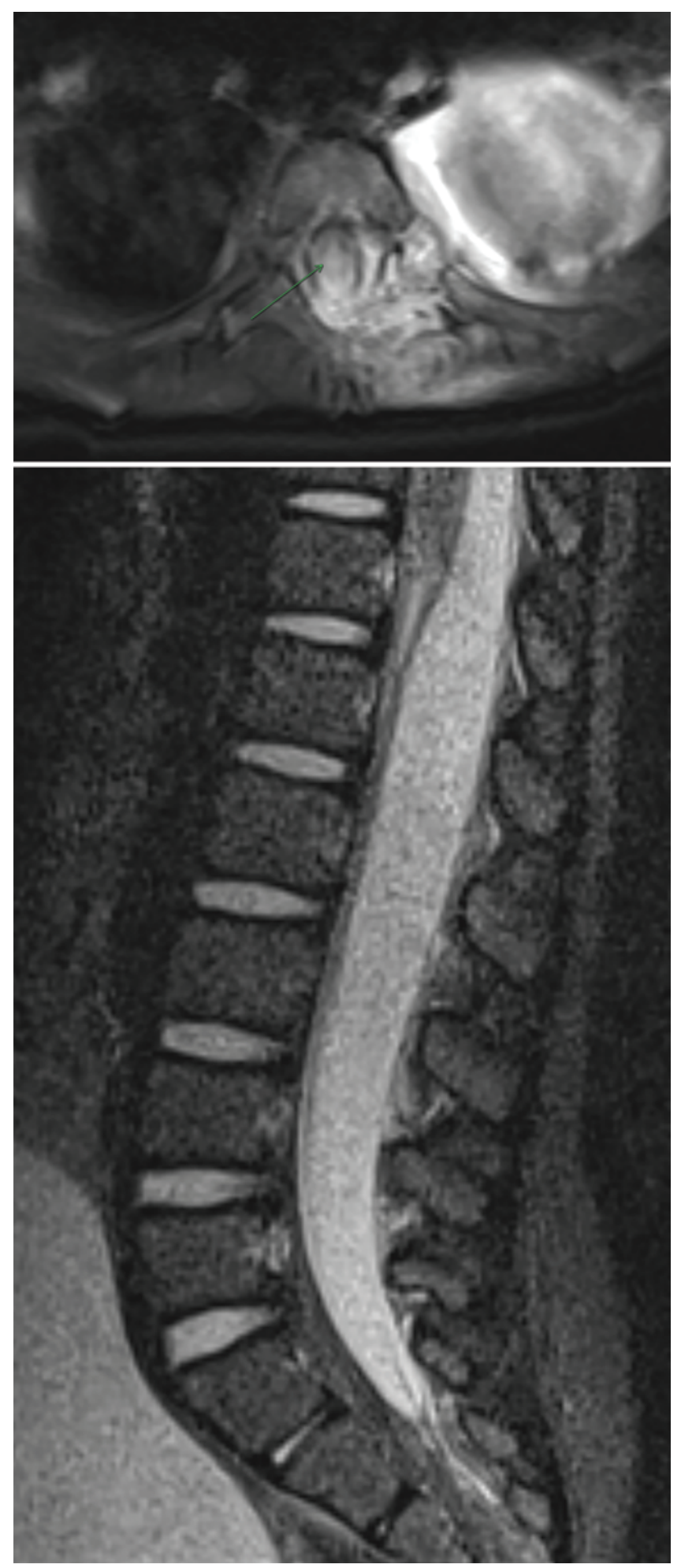

FIG. 2. Upper: Axial MRI T2 STIR sequence obtained at T-3, demonstrating intramedullary T2 hyperintensity suggestive of a spinal cord contusion (green arrow). Lower: Sagittal T2 STIR sequence of the lower thoracic and lumbar spine depicting a large subdural fluid collection with anterior displacement of the lower thoracic spinal cord and conus. Imaging characteristics on T1- (not shown) and T2-weighted imaging were consistent with CSF. The clinical significance of this hygroma was unknown. Lumbar puncture was offered for drainage, but the family declined this intervention. Figure is available in color online only. 
of fibrin glue (Tisseel, Baxter International) under the T-2 lamina via a 20 -gauge, $9-\mathrm{cm}$ spinal needle, and this provided sufficient coverage of both dural defects at T-2 and T-3 (Fig. 4).

\section{Posttreatment}

Postprocedure, her chest tube output was 0 and $8 \mathrm{ml}$ on hospital Days 11 and 12, respectively. She remained asymptomatic, and serial radiographs were stable without
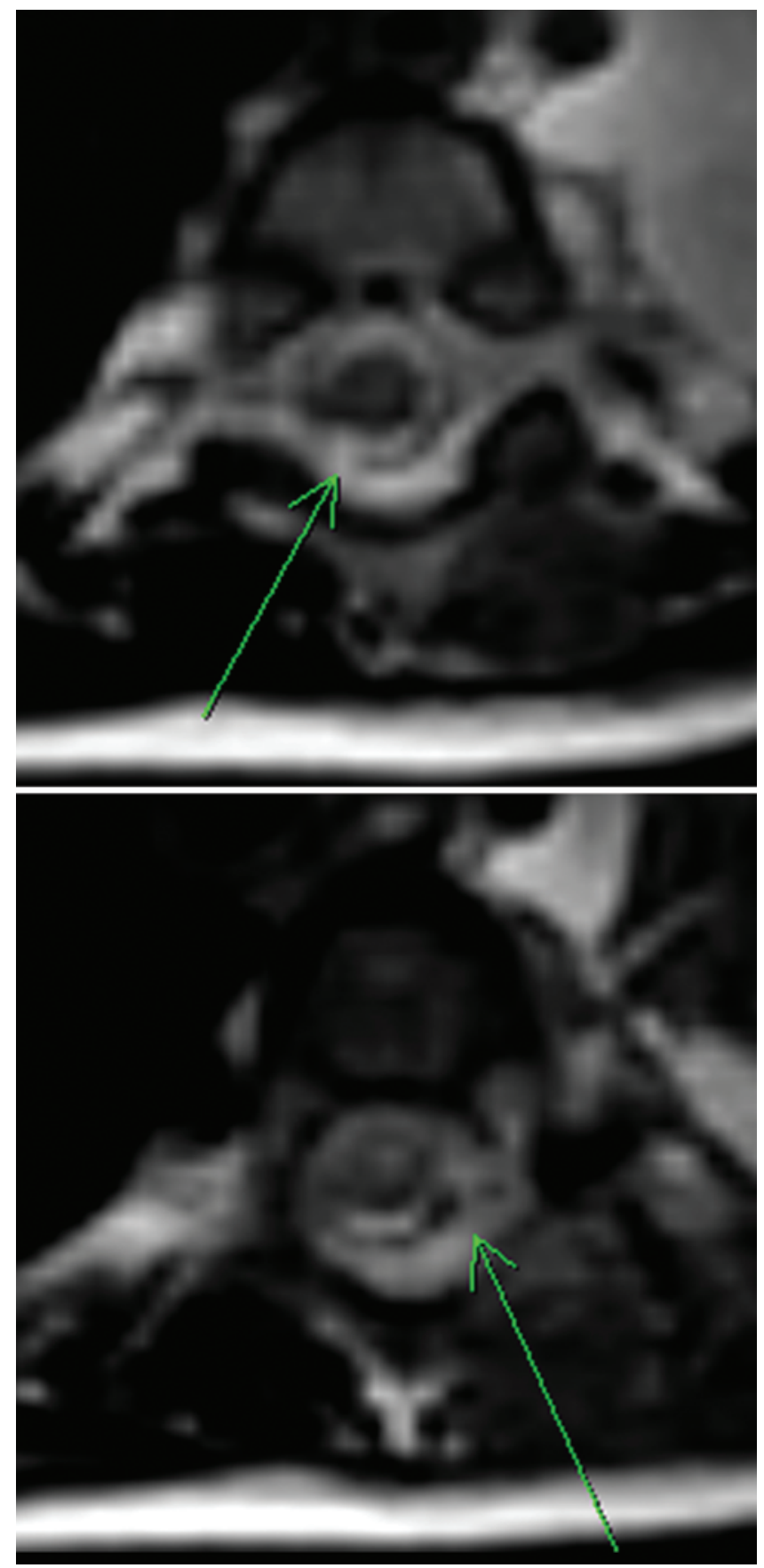

FIG. 3. Reformatted axial thoracic CISS MRI sequences showing areas of CSF leakage (arrows) from a right posterolateral dural defect at the level of T-2 (upper) and a left lateral dural defect at T-3 (lower). Figure is available in color online only. reaccumulation of pleural fluid. Her thoracostomy tube was removed on Day 12, and she was transferred to inpatient rehabilitation on hospital Day 13. She completed a 6-week inpatient rehabilitation course without any pulmonary or neurological complications. At the time of discharge from rehabilitation, her neurological status remained an ASIA Grade A. She was seen as an outpatient at 9 weeks postprocedure with an unchanged neurological exam. Chest radiograph did not show recurrence of the pleural effusion and suggested closure of the SPF. Additionally, there was no evidence of thoracic kyphosis as a result of the left T-3 pedicle and laminar fractures, and she will continue to be managed conservatively.

\section{Discussion}

Subarachnoid-pleural fistulas are unique clinical entities that can develop with disruption of both the parietal pleura and the thoracic dura. ${ }^{8}$ The 2 most common scenarios in which they are encountered are severe thoracic trauma or iatrogenic injury during anterolateral approaches to the thoracic spine. Given the rarity of these fistulas, their diagnosis requires a high index of suspicion from clinicians. Symptoms of SPF stem from hydrothorax (dyspnea, hypoxia, respiratory distress) or intracranial hypotension (postural headache, altered mental status, nausea and vomiting, diplopia, auditory abnormalities, and even chronic cerebellar hemorrhage ${ }^{22}$ ). Additional clinical signs can include recurrent pleural effusion, transudative effusion, abnormally high thoracostomy tube output, and imaging findings consistent with intracranial hypotension. Pleural fluid positive for $\beta 2$-transferrin increases diagnostic confidence as this test reaches $100 \%$ sensitivity and $95 \%$ specificity for the presence of CSF. ${ }^{23}$ While CT myelography is the imaging modality of choice to visualize SPFs, MRI with thin-section T2-weighted sequences (such as CISS) and contrast-enhanced cisternography have become reasonable alternatives as they have been reported to have $76 \%$ and $100 \%$ sensitivity, respectively, in detecting CSF leaks. ${ }^{2}$

Optimal treatment for SPF remains controversial; however, there is consensus that such fistulas are unlikely to resolve spontaneously. ${ }^{7}$ The intrathoracic pressure ranges from -5 to $-7.5 \mathrm{~cm} \mathrm{H}_{2} \mathrm{O}$, whereas the intradural pressure is between +10 to $+15 \mathrm{~cm} \mathrm{H}_{2} \mathrm{O}$; thus, this pressure gradient plays a critical role in maintaining fistula patency. ${ }^{20}$ Surgical intervention is often required to eliminate this pressure differential and entails direct repair of pleural and dural defects, either with open surgery or in a minimally invasive fashion..$^{10,20}$ This surgical intervention most commonly requires the use of a dural substitute or sealant (Tisseel, DuraSeal) and can be augmented with a vascularized graft.7,8,19 Other authors have reported success with secondary occlusion of fistulas by using an epidural blood patch (EBP) ${ }^{1}$ or synthetic material such as DuraSeal, Tisseel, and even Onyx; ;,6,11 however, all of these successes occurred in adult patients. Kurata et al. reported that 5-14 days of continuous noninvasive positive pressure ventilation (NIPPV) can be used as an adjunct to chest tube drainage, with the goal of increasing intrathoracic pressure and reducing the pressure gradient between the 

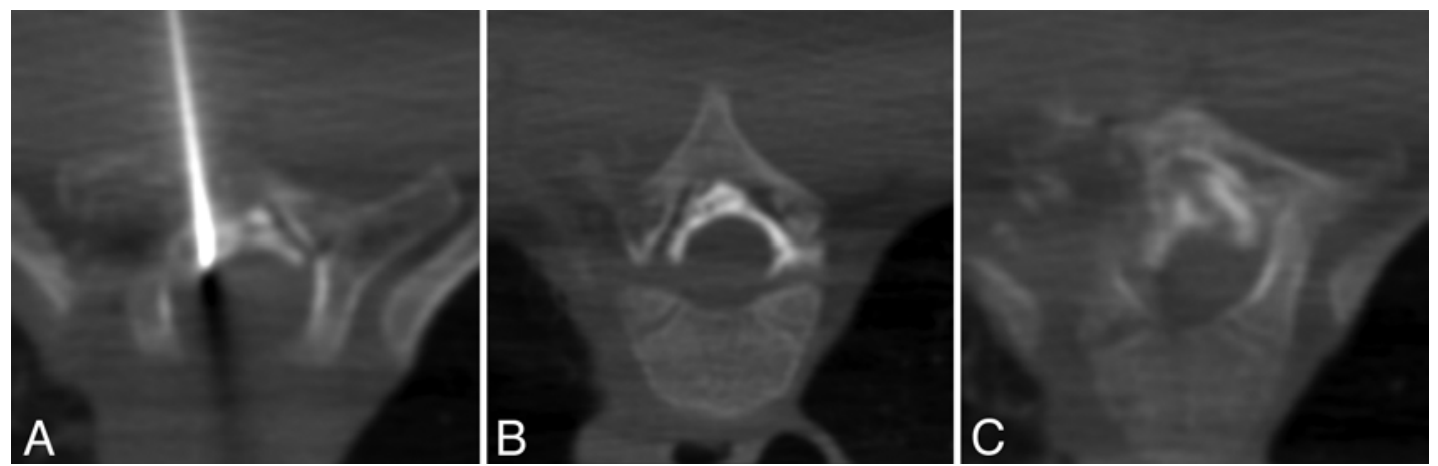

FIG. 4. Computed tomography-guided percutaneous epidural injection of $2 \mathrm{ml}$ of fibrin glue (Tisseel) under the lamina of T-2 (A). The fibrin glue can be visualized as a hyperdensity in the dorsal epidural space spanning the dural defects at T-2 (B) and T-3 (C). This technique was successful in occluding the SPF.

pleural and subarachnoid spaces. ${ }^{12}$ In the pediatric population, continuous NIPPV may be a therapeutic challenge as patient compliance can be difficult and places the patient at risk for side effects of continuous NIPPV (skin breakdown, gastric distention, dry mouth). Furthermore, there is only 1 documented pediatric case of PPV as therapy for SPF; however, the 9-month-old patient in that case required intubation and invasive PPV by endotracheal tube for nearly 2 weeks. ${ }^{24}$

There are few reports of SPF in the pediatric neurosurgical literature, with sporadic single cases that mainly occur after thoracic tumor resection and trauma., $, 10,18,24$ Common to these cases were requirements for open surgery and repair of the dural defect. Postoperatively, patients in these cases remained in the hospital for 7-14 days for observation and chest tube management. Liang et al. reviewed 40 cases of traumatic SPF occurring between 1959 to 2005 and reported 18 cases of SPF in pediatric patients. ${ }^{13}$ Of these, 7 cases (age range 3-18 years) were secondary to penetrating trauma, and all patients in these cases required intervention in the form of a chest tube or open repair. We were unable to find any cases of spontaneous resolution of SPF in the pediatric population. Reports discussed above are summarized in Table 1.

Our patient represents the youngest reported case of penetrating traumatic SPF and the first report of percutaneous epidural injection of fibrin glue alone for the successful treatment of SPF in a pediatric patient. The abrupt reduction in chest tube output and the serial images that showed no reaccumulation of her pleural effusion allowed for the removal of her chest tube on postoperative Day 2. In this case, we believe the percutaneous epidural technique was advantageous as it obviated the need for open surgery and reduced the chest tube duration and postoperative hospital stay, enabling timely transfer to inpatient rehabilitation.

We do not believe the bullet traversed the spinal cord and venture that dural lacerations were probably attributable to impact from osseous fragments against the thecal sac or secondary shockwave from the blast injury. Interestingly, the dural defect at T-2 was located on the right posterolateral aspect of the cord, at a distance from the bullet trajectory and left pedicle fractures. Given that our patient had multiple, isolated dural defects, we speculate that her dura was probably very friable, even possibly "shredded," with smaller defects impossible to visualize by the CISS MRI sequence. Therefore, we do not believe that shredded dura with multiple small defects would be a contraindication for CT-guided percutaneous treatment for SPF-in fact, it may be an ideal first-line treatment for multiple dural defects that would be difficult to access via a single surgical incision.

Our patient presented with a complete spinal cord injury and did not display evidence of spinal canal stenosis; therefore, mass effect from percutaneous fibrin glue injection was less concerning, especially when a small volume $(2 \mathrm{ml})$ of fibrin glue was injected to cover 2 spinal levels. We can only speculate that large volumes may increase the risk. In cases of incomplete spinal cord injury or in those with critical canal stenosis, the percutaneous addition of material into the epidural space may place the patient at risk for neurological decline. Close postprocedure monitoring would be required, and surgical decompression may be necessary if decompensation occurs. Certainly, if a patient had an unstable middle column injury with retropulsed fragments resulting in canal stenosis and an incomplete injury, surgical intervention rather than the use of percutaneous techniques would be more appropriate treatment.

Our decision to use fibrin glue over an alternative material, such as an EBP, partially stems from surgeon preference, and we do not have extensive experience with EBP. To our knowledge, no studies have compared targeted EBP and fibrin glue to suggest the superiority of one over the other. Despite representing the gold standard for post-lumbar puncture headaches, EBP has a widely variable success rate and a failure rate up to $44 \%$ and often requires multiple treatments when used for spontaneous intracranial hypotension. ${ }^{4,21}$ The largest case series involving percutaneous epidural fibrin glue injection involved 23 patients and reported a $65 \%$ success rate..$^{16}$

In pediatrics, EBP requires weight-based dosing, and typically between 0.3 and $0.4 \mathrm{ml} / \mathrm{kg}$ is used and larger doses (up to $0.8 \mathrm{ml} / \mathrm{kg}$ ) have been reported for repeat procedures. ${ }^{3,5}$ Our patient's weight was approximately $15 \mathrm{~kg}$, and thus between 4.5 and $6 \mathrm{ml}$ of blood would have been used. This is roughly $2-3$ times the volume of fibrin glue that was injected. As discussed above, whether a larger volume of material injected into the epidural space would be problematic is subject to speculation. 


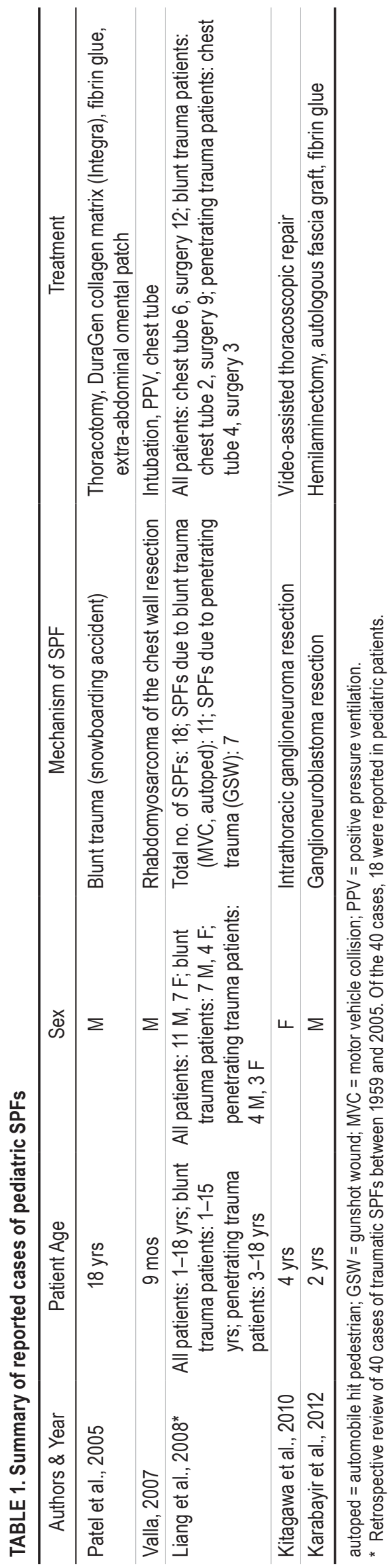

Given the increasing popularity of CT-guided epidural patching for CSF leaks, we agree with previous authors that it represents a viable alternative to open surgery., $1,6,17$ While this technique may not be applicable for all patients, future studies may identify factors that better stratify patients toward this option. For example, Mihlon et al. demonstrated that CT-guided percutaneous EBP is more likely to be successful with a durotomy size less than $5 \mathrm{~mm} .^{14}$

\section{Conclusions}

Our case represents the youngest pediatric patient with a penetrating traumatic SPF successfully treated with CT-guided percutaneous epidural injection of fibrin glue alone. Our case demonstrates that the CT-guided percutaneous epidural technique can be safely used in pediatric patients and in patients with multiple dural defects and can offer patients a shorter hospital stay if fistula closure is achieved. Factors determining the success rate of the percutaneous obliteration of SPFs require investigation.

\section{References}

1. Agarwal V, Sreedher G, Rothfus WE: Targeted CT-guided epidural blood patch for treatment of spontaneous intracranial hypotension due to calcified intradural thoracic disc herniation. Interv Neuroradiol 19:121-126, 2013

2. Algin O, Hakyemez B, Gokalp G, Ozcan T, Korfali E, Parlak M: The contribution of 3D-CISS and contrast-enhanced MR cisternography in detecting cerebrospinal fluid leak in patients with rhinorrhoea. Br J Radiol 83:225-232, 2010

3. Borges BC, Wong G, Isaac L, Hayes J: Unusual presentation of postdural puncture headache requiring repeat epidural blood patch in a 4-year-old child. Paediatr Anaesth 24:541543,2014

4. Chai CM, Banu MA, Cobb W, Mehta N, Heier L, Boockvar JA: Novel hydrogel application in minimally invasive surgical approaches to spontaneous intracranial hypotension. Report of 2 cases. J Neurosurg 121:976-982, 2014

5. Franklin AD, Hays SR: Successful management of a thoracic cerebrospinal fluid cutaneous fistula in a two year old child using a thoracic epidural blood patch. J Clin Anesth 25:331334, 2013

6. Gladstone JP, Nelson K, Patel N, Dodick DW: Spontaneous CSF leak treated with percutaneous CT-guided fibrin glue. Neurology 64:1818-1819, 2005

7. Heller JG, Kim HS, Carlson GW: Subarachnoid-pleural fistulae-management with a transdiaphragmatic pedicled greater omental flap: report of two cases. Spine (Phila Pa 1976) 26:1809-1813, 2001

8. Hentschel SJ, Rhines LD, Wong FC, Gokaslan ZL, McCutcheon IE: Subarachnoid-pleural fistula after resection of thoracic tumors. J Neurosurg 100 (4 Suppl Spine):332-336, 2004

9. Karabayir N, Demirkol D, Al IO, Dolas I, Sencer A: Subarachnoid-pleural fistula in a child: the cause and treatment. Pediatr Int 54:929-931, 2012

10. Kitagawa RS, Satyan KB, Relyea K, Dauser RC, Nuchtern JG, Minifee PK, et al: Video-assisted thorascopic repair of a subarachnoid-pleural fistula in a child after thoracic tumor resection: technical note. Spine (Phila Pa 1976) 35:E347E350, 2010

11. Knafo S, Parker F, Herbrecht A, Court C, Saliou G: Percutaneous treatment of subarachnoid-pleural fistula with Onyx. J Neurosurg Spine 18:378-381, 2013

12. Kurata Y, Yoshimoto M, Takebayashi T, Kawaguchi S, Yamashita T: Subarachnoid-pleural fistula treated with noninva- 
sive positive pressure ventilation: a two-case report and literature review. Spine (Phila Pa 1976) 35:E908-E911, 2010

13. Liang MK, Moore EE, Williams AM, Marks JL: Traumatic subarachnoid pleural fistula. J Trauma 65:1155-1161, 2008

14. Mihlon F, Kranz PG, Gafton AR, Gray L: Computed tomography-guided epidural patching of postoperative cerebrospinal fluid leaks. J Neurosurg Spine 21:805-810, 2014

15. Milloy FJ, Correll NO, Langston HT: Persistent subarachnoid-pleural space fistula; report of a case. J Am Med Assoc 169:1467, 1959

16. Patel MR, Caruso PA, Yousuf N, Rachlin J: CT-guided percutaneous fibrin glue therapy of cerebrospinal fluid leaks in the spine after surgery. AJR Am J Roentgenol 175:443-446, 2000

17. Patel MR, Louie W, Rachlin J: Postoperative cerebrospinal fluid leaks of the lumbosacral spine: management with percutaneous fibrin glue. AJNR Am J Neuroradiol 17:495-500, 1996

18. Patel MR, Wehner JH, Soule WC, Meter JJ: Intracranial hypotension and recurrent pleural effusion after snow-boarding injury: a manifestation of cerebrospinal fluid-pleural fistula. Spine J 5:336-338, 2005

19. Raffa SJ, Benglis DM, Levi AD: Treatment of a persistent iatrogenic cerebrospinal fluid-pleural fistula with a cadaveric dural-pleural graft. Spine J 9:e25-e29, 2009

20. Sahota S, Nassr A, Khan MH, Marsh RW, Moran SL, Arnold PM, et al: Treatment of a thoracic dural-pleural fistula with a vascularized omental flap: a case report. Spine (Phila Pa 1976) 37:E683-E685, 2012

21. Schievink WI, Maya MM, Louy C, Moser FG, Sloninsky L:
Spontaneous intracranial hypotension in childhood and adolescence. J Pediatr 163:504-510, 2013

22. Schievink WI, Maya MM, Nuño M: Chronic cerebellar hemorrhage in spontaneous intracranial hypotension: association with ventral spinal cerebrospinal fluid leaks: clinical article. J Neurosurg Spine 15:433-440, 2011

23. Skedros DG, Cass SP, Hirsch BE, Kelly RH: Beta-2 transferrin assay in clinical management of cerebral spinal fluid and perilymphatic fluid leaks. J Otolaryngol 22:341-344, 1993

24. Valla FV: Subarachnoid-pleural fistula in an infant treated with mechanical positive-pressure ventilation. Pediatr Crit Care Med 8:386-388, 2007

\section{Disclosure}

The authors report no conflict of interest concerning the materials or methods used in this study or the findings specified in this paper.

\section{Author Contributions}

Conception and design: Chern. Acquisition of data: Chu. Analysis and interpretation of data: Chu. Drafting the article: Chu. Critically revising the article: Chu, Miller, Chern. Reviewed submitted version of manuscript: all authors. Study supervision: Chern.

\section{Correspondence}

Jason Chu, Department of Neurosurgery, Emory University School of Medicine, 1365-B Clifton Rd., Ste. 6200, Atlanta, GA 30322. email: jason.chu@emory.edu. 